\title{
Article
}

\section{End-of-life care guidelines and care plans in the intensive care unit}

\author{
Luckett, Alison
}

Available at http://clok.uclan.ac.uk/17185/

Luckett, Alison (2017) End-of-life care guidelines and care plans in the intensive care unit. British Journal of Nursing, 25 . ISSN 0966-0461

It is advisable to refer to the publisher's version if you intend to cite from the work.

For more information about UCLan's research in this area go to http://www.uclan.ac.uk/researchgroups/ and search for < name of research Group>.

For information about Research generally at UCLan please go to http://www.uclan.ac.uk/research/

All outputs in CLoK are protected by Intellectual Property Rights law, including Copyright law. Copyright, IPR and Moral Rights for the works on this site are retained by the individual authors and/or other copyright owners. Terms and conditions for use of this material are defined in the policies page.

\section{CLoK}

Central Lancashire online Knowledge www.clok.uclan.ac.uk 


\title{
End-of-life care guidelines and care plans in the intensive care unit
}

\author{
Alison Luckett
}

\begin{abstract}
The aim of this literature review was to examine end of life (EoL) care practice within the Intensive care unit (ICU). By exploring the literature, it is hoped to suggest how national guidance can be integrated into the ICU setting. Delivering high-quality, patient-centred EoL care is high priority on the UK health agenda. The highly technological environment within ICU can create barriers to recognising death and initiating EoL care planning. Despite recommendations in the literature for the integration of standardised guidance, implementation, compliance and evaluation are yet to be widely reported. This literature review highlights the need for ICU health professionals to embrace EoL guideline recommendations. Development of robust processes is vital to inform future practice.
\end{abstract}

Key words: Intensive care units End of life care Decision making - Documentations

\section{a personalised care plan for current and future support and treatment.'}

NICE, 2011

The Liverpool Care Pathway (LCP) was used as primary guidance for EoL care within the UK until July 2014 when following intense media scrutiny which sparked national review it was withdrawn (Compassion in Dying, 2015). Despite being widely used in community and ward settings in the UK and overseas, it was never designed specifically for ICU (Handy et al, 2013). Walker and Read (2010) found little evidence that the later adapted LCP for ICU had been widely adopted. It too was withdrawn concurrently with the general LCP in 2014.

\section{Background}

Recommendations for high-quality EoL care from the Leadership Alliance for the Care of Dying People (LACDP) (2014) include timely recognition of dying, sensitive communication, the involvement of the patient and family in care decisions, supporting those involved and forming an individualised care plan to encompass compassionate care. These are broad generic terms and may leave too much room for interpretation when adopted in an ICU.

Prognostic uncertainty in critically ill patients often results in EoL decisions being made too late, preventing timely recognition and individualised care planning (Campbell, 2013; Nelson, 2006; Hamric and Blackhall, 2007). The adoption of advanced care planning for EoL is rare within ICU as most patients are haemodynamically unstable when they arrive and priority is given to restoring homeostasis, treatment and cure. Conversations with patients and their families or carers on admission often focus on treatment aims rather than discussion of EoL preferences should this be necessary.

The majority of deaths in the ICU occur after a decision to withdraw or withhold treatment once consensus has been reached agreeing the patient has no chance of survival (Morgan, 2008; Dean et al, 2010). Curtis et al (2010) highlight the increase in older patients presenting to ICU following cardiopulmonary resuscitation with pre -existing co morbidities. These patients may be for limited interventions, with agreement for transition to EOL care if unsuccessful (Monkhouse 2013; Morgan et al, 2014). The use of targeted therapies where escalation of care is not recommended and more patients being transferred from ICU for EoL care requires ICU clinicians to initiate EoL care planning, adhering to policies and guidelines. Checkley et al (2014) found an annual ICU mortality rate of $10.9 \%$ over
Alison Luckett, Nurse, Lecturer Pre-registration Nursing, University of Central Lancashire, aluckett1@uclan.ac.uk

Accepted for publication: February 2017 


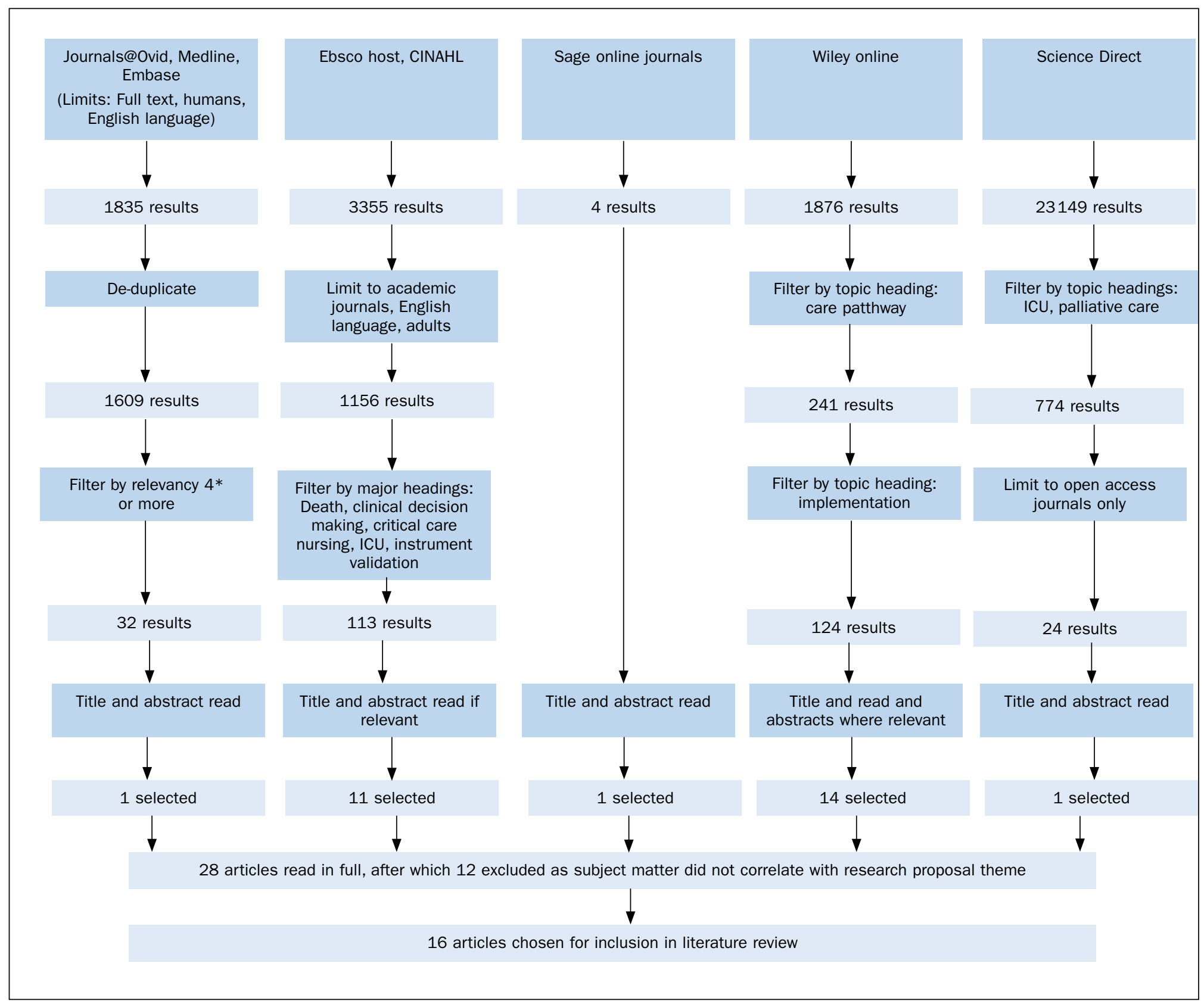

Figure 1. Search strategy flowchart

69 centres in the US. Capuzzo et al (2014) found European mortality rates within ICU were $19.1 \%$ with a further $4.8 \%$ of post-ICU patients dying prior to discharge. Therefore recognising EoL care as a legitimate outcome within the ICU could ensure patient and family choices at EoL are discussed and achieved when possible.

\section{Aim}

The aim of this international literature review was to gain $\Xi$ understanding of EoL care practices in the ICU, to ascertain the guidelines in use and how care is documented. Sprung et al (2003) reported the multi-centre Ethicus study which found significant religious, cultural and procedural variation in European EoL practices. Since then EoL care and guidelines have changed. While this study examined practices it did not specifically examine documentation used or guidelines followed. Ethicus II is currently being undertaken internationally to examine current practice with an aim to inform policy makers and assist in improving EoL care (Bassford, 2015).

\section{Methods}

To understand the complex issues when planning care for dying patients in ICU a literature review was undertaken (Figure 1). The MeSH terms 'end of life', 'dying', 'intensive care' and 'care planning' have were used and Boolean operators 'and, or' were used. Publication years 2008 to 2015 were chosen as this period represents the emergence of EoL care as a priority within healthcare following the publication of the End of Life Care Strategy (DH, 2008). Multiple databases were searched, EBSCOhost, Ovid, Medline, CINAHL, in addition publishing 
Table 2. Literature search results

\section{Author/s (year of publication)}

Aslakson et al (2014)

Bjurling-Sjöberg et al (2015)

Chan and Webster (2013)

Coombs and Long (2008)

Coombs et al (2012)

Cox et al (2012)

Efstathiou and Walker (2014)

Glavan et al (2008)

Langley et al (2014)

Morgan (2008)

Penrod et al (2012)

Ranse et al (2015)

Ryan and Seymour (2013)

Ramasamy Venkatasalu et al (2015)

Qualitative semi-structured interviews

UK

\section{Literature review \\ UK}

Quantitative, prospective multisite observational study of palliative care processes USA

Qualitative, cross-sectional survey

Australia

\section{Literature review}

UK

\section{Discussion paper}

UK

Watts (2013)

Literature review

UK

\section{Key recommendations/conclusions}

Using US national ICU guidelines can help to improve palliative care

Implementation of care plans in ICU rely on multifaceted strategies, committed individuals and bottom-up approach

Evidence supporting impact of EoL care plans is lacking. Audit and evaluation is required

EoL care plans for ICU must be a blend of policy guidance and clinical process

Adopting EoL care plans may be inhibited when the greatest challenge in ICU is diagnosing dying

EoL protocols specific to ICU can help provide excellent care

Practice guidelines for EoL care in ICU are needed to reduce ambiguity

Retrospective analysis of medical notes of dying patients provides quality markers for improving EoL care

Use of formal guidelines and education recommended to improve EoL care and communication

ICU nurses require educational support to implement EoL care guidance practice

Use of a care and communication bundle approach was applied inconsistently and infrequently

Identification of factors influencing EoL care provision in order to develop a standardised order for care

Use of guidelines can help reduce emotiona stress for ICU nurses caring for patients at EoL

Staff still require clear guidelines for EoL care in ICU despite the withdrawal of the LCP

EoL care plans exist as a means of documentation but offer little help in complex decision making

EoL care pathways in general should be welcomed as a means to enhance care

\section{Strengths/limitations}

None identified

Mixed methods

Limited dataset, retrospective data collection, risk of recall bias

None identified

None identified

Convenience sampling, study bias, poor transferability

None identified

Single site self-selected interviewees could limit transferability

Limited variables measured, single region, low response rate $(41.2 \%)$, recall bias

Reasonable response rate (67\%)

Sample bias as self-selecting, poor generalisability

None identified

Only three sites used retrospective medical record analysis relied on accurate documentation

Convenience sampling, poor response rate $(45 \%)$

None identified

First study to explore the issues following LCP withdrawal Small sample size limits transferability

None identified

None identified websites were also searched including Wiley, ScienceDirect and Sage Journals online. Further limiters as shown in Figure 1, were used when appropriate depending on the search engine for each source. Choosing such broad search terms resulted in 
a high volume of worldwide literature from which titles and key words were read to ascertain relevancy. Appropriate filters facilitated reduction of the results to a reasonable amount of abstracts to be read. A total of 28 articles were read in full and 12 excluded as their focus did not meet the aims of the research proposal. Reasons for exclusion of the articles involved those focusing on ethical decisions around withdrawal of treatment, relationship between EoL care planning and reduced length of stay and articles examining advanced care planning or quality of life scoring systems.

\section{Results}

A total of 16 articles were deemed relevant and chosen for inclusion in the review (Table 1). The decision to take such a broad international literature search from multiple sources is drawn from previous experience of conducting a literature review concentrating on UK EoL practices in the ICU. The aim of the literature review was to explore if and how LACPD (2014) guidance could be adopted in ICU following the withdrawal of the LCP for dying patients. Restricting the review to UK sources only within the same publication years (2008-2015) yielded very low results, perhaps reflective of the lack of UK based research in EoL within ICU. No studies were found suggesting the new LACPD (2014) guidance had been adopted or detailing what care plans were in use. It may have been too early to evaluate the effects of the guidance as new care plans may still have been in development, or it may be that new guidelines had not been adopted within the specialist setting of ICU.

\section{Discussion}

Review of the literature resulted in several themes emerging in relation to EoL practices in the ICU setting. These include difficulties with integration of national frameworks, decision making, care planning, implementation and compliance, and evaluation.

\section{Integration of national frameworks}

Coombs and Long (2008) looked at whether national guidelines can assist in providing a good death within ICU. While it is acknowledged that they provide a broad overview of key areas, they fail to address the complexities of ICU care. Specific barriers within ICU are identified with regard to the decision to change the focus of care from curative to EoL. Therefore EoL care documents developed for ICU must be appropriate to setting and context.

Morgan (2008) stated that national EoL policy for the ICU is imprecise and integration of hospital wide EoL care is often absent from the ICU setting.

This absence of formal national guidelines being used in ICU areas is highlighted in qualitative research undertaken ङ by Efstathiou and Walker (2014) and Venkatasalu et al (2015). Both articles reported on semi-structured interviews regarding provision of EoL care in the ICU. Although undertaken in different UK locations, none of the ICUs had any formal guidance or standardised care plans. Both articles recommended that the development of standardised guidelines within ICU at
EoL could help reduce ambiguity in relation to withdrawal of treatment processes and prompt discussion to facilitate patient and family choices.

The use of an EoL care plan as a vehicle to prompt dialogue for individual choice is further endorsed by Watts (2012) who suggested that conversations about EoL preferences should occur sooner in the dying trajectory. Aslakson et al (2014) supported early identification of EoL needs but reported that results from a randomised controlled cluster trial found that using standardised EoL care plans had no effect on quality care outcomes (Curtis et al, 2011)

In comparison, Chan and Webster (2013) published an update of a Cochrane systematic review concluding that recommendation to use EoL care plans could not be made, stating a lack of direct high-quality evidence supporting their effectiveness. They recommended that independent audits of care plans are required to build the evidence base. Since this Cochrane systematic review there has been another review (Chan et al, 2016). Although outside the limits of the literature review, the Chan et al (2016) concluded that there was limited evidence available to draw conclusions on the effectivness of EoL care pathways.

\section{Identifying EoL and decision making}

A significant barrier identified in the literature for EoL care in ICU centred on the difficulties in diagnosing when a patient is nearing EoL. Coombs et al (2012) undertook qualitative research through semi-structured interviews of ICU doctors and nurses. They identified the complex variables evident when diagnosing dying in ICU. Complex variables include prognostic uncertainty, defining futility, reaching a consensus in line with the values and beliefs of all stakeholders and managing transition to EoL care.

Differentiating between treatments prolonging death or enabling recovery is not straightforward (Ryan and Seymour, 2013). The biomedical culture of dying in hospital perceives death as a failure, favouring technological interventions as an avoidance tactic (Watts, 2012). This combined with a lack of knowledge on how clinicians diagnose dying inhibits timely EoL discussion. There are also inconsistencies of predictive tools developed for probability of dying in ICU, leading to diagnosis of dying being considered too late to initiate EoL care planning (Cox et al, 2012). Therefore it could be argued that because timely diagnosis of EoL is the first priority from LACPD (2014) guidance for high-quality EoL care, integrating this principle into the ICU setting is potentially flawed from the outset.

Once the decision has been reached to progress to EoL care, RamasamyVenkatasalu et al (2015) advocated that having specific EoL guidelines in ICU are vital to reduce inconsistencies in care. In their study RamasamyVenkatasalu et al (2015) looked at the effects following the withdrawal of the adapted LCP-ICU in two large acute hospitals in the UK. Interviews uncovered concerns that without this evidence-based guidance clinicians became unsure of the action to take, nurses became more reliant on doctors for treatment decisions and concerns rose that there was no robust documentation to safeguard practice. 


\section{Personal distress}

The effects of personal distress to clinicians experienced by lack of guidance are further explored by Coombs and Long (2008), Ryan and Seymour (2013) and Efstathiou and Walker (2014). Coombs and Long (2008) discuss the difficulties arising between doctors and nurses when deciding the process of withdrawal of treatments. Differences in values, beliefs and opinion between the disciplines caused difficulties achieving consensus. They surmise that evidence based EoL guidelines developed from UK policy could help to reduce this (Coombs and Long, 2008). Ryan and Seymour (2013) specifically examined the emotional stress ICU nurses face when initiating EoL care. They found that once a doctor made the decision that active treatment should stop, nurses bear the responsibility for stopping them. The nurse is the main facilitator of treatment withdrawal (Ranse et al, 2014). A lack of clear guidance and consensus on the process can lead to increased distress for nurses. Efstathiou and Walker (2014), conducted semi structured interviews of nurses providing EoL care in ICU at a single site UK hospital. The lack of standardised evidence-based EoL care guidelines in the setting was found to increase uncertainty, nurse dissatisfaction and inhibit effective communication.

\section{Documentation and processes}

Several of the articles included in the review highlight the need for a clear documentation process owing to variations in EoL care and withdrawal of treatment decisions. Venkatasalu et al (2015) undertook a qualitative study of how the transfer to generic EoL guidance in ICU was affecting care. They found inconsistencies and communication difficulties were arising owing to adopting these care plans following the withdrawal of the LCP-ICU. It was no longer clear why care was either given or not given. Recommendations from the study include development of ICU-specific guidance to include shared decision making and staff education. Langley et al (2014) undertook a cross-sectional survey of South African ICU nurses' views and experiences of the process of withdrawing treatment. It was surmised that development of formal guidelines could improve inter-professional and family communication at EoL.

Cox et al (2012) stated that although treatment withdrawal decisions should be made on benefit versus burdens basis; owing to variations and differing interpretation of the decisions to stop specific therapies, robust documentation is vital. Although Ryan and Seymour (2013) highlighted the lack of research evidence supporting specific ICU EoL guidelines, they acknowledged their potential for standardising care and communication.

Coombs and Long (2008) suggested a lack of evidence behind decisions to limit or withdraw specific treatments for dying patients such as mechanical ventilation. Accurate ICUspecific EoL documentation could help build the evidence base for structured withdrawal of treatment processes.

While the argument for standardised and robust documentation is evident in the literature, evidence regarding its effectiveness is variable. Aslaskon et al (2014) cited a large multi-centre randomised controlled trial in the US where standardised EoL care protocols were used (rather than non-standardised ones) (Curtis et al, 2011).The study found no perceived improvement in quality outcome measures of dying by nurses and families. ICU nurses rely more on experience to guide practice in delivering high-quality care at EoL than guidelines (Ryan and Seymour,2013). Without standardised guidance junior nursing and medical staff in ICU will lack the knowledge to provide quality care (RamasamyVenkatasalu,et al, 2015). Increased staff turnover and recruitment of junior nursing staff could be a significant factor within ICU settings in the UK.

\section{Implementation and compliance}

Difficulties with implementation and compliance with standardised care plans is explored within the literature. BjurlingSjöberg et al (2015) undertook an exploratory mixed-methods study examining how standardised clinical care plans are used in Swedish ICUs. Multiple factors were identified, the format had to be user-friendly and have perceived benefits for staff and patients. Bottom-up initiatives were deemed to be more successful with repeated reminders to use the care plans required to help embed new working practices.

Lack of involvement by doctors was found to be a barrier with implementation and compliance. Without structured guidelines ICU nurses over-rely on doctors for decision making when caring for dying patients (Venkatasalu et al, 2015). Bjurling-Sjöberg et al (2015) suggested that a reason for doctor's reluctance to use standardised care plans is the perception that they lose control over decision making. Further research could identify other significant factors affecting implementation and compliance. Watts (2013) discussed challenges with implementation and sustainability when using EoL care plans, with transient workforce, organisational culture, role boundaries and concern about litigation as mitigating factors. In addition there are concerns around whether standardised care plans satisfy the recommendation for individualised patient care at EoL (Watt, 2013). Formulating an individualised plan of care is one of the five key recommendations within the revised LACDP (2014) guidance.

\section{Evaluation}

Several articles examined the importance of evaluation. Penrod et al (2012) undertook a multisite observation study evaluating the performance of key EoL care processes in the ICU.The use of and compliance to a national EoL care bundle within the US was examined.They found that evidence-based care processes were frequently missed and the care plans not consistently followed. However one of the limitations of this study is that was a retrospective case analysis and there is potential for the care to have been given but not documented.

In comparison, Glavan et al (2008) suggest that retrospective case note analysis when evaluating EoL care provides valuable information to guide quality improvement. How the effect of a standardised care plan is evaluated can have a profound effect on its perceived usefulness (Bjurling-Sjöberg et al, 2015). Therefore when undertaking such research it is important to ensure robust methods and data analysis are adopted.

\section{Limitations}

Limitations of the research presented in this literature review are that it is largely based on participant views or retrospective 
case note analysis, rather than observation in practice (Penrod et al, 2012; Ramasamy Venkatasalu et al, 2015). Observation in practice poses moral and ethical challenges for researchers that are difficult to overcome in EoL situations. EoL care plans should be independently audited and their subsequent use evaluated despite the methodological challenges posed by research around EoL (Chan and Webster, 2013).

\section{Implications and recommendations}

Despite an extensive international literature search, only 16 articles were found in relation to the use of standardised EoL care plans in the ICU. The inconsistencies and ambiguity regarding how EoL care is performed and documented is evident within the literature. Despite acknowledgement within the articles presented that the evidence base for the use of EoL care plans in ICU lacking, emphasis is placed on recommending the need for their development and evaluation. To date it would appear that nationally no research has taken place into ICU EoL care plans introduced under the new LACPD (2014) guidance. The effectiveness of individualised care planning and guideline development in assisting health professionals to manage EoL care within ICU requires further research.

\section{Conclusion}

Recognising EoL in a timely manner may always be difficult for health professionals owing to the unpredictable clinical course of illnesses in ICU. Providing high-quality EoL care is an important skill that ICU nurses need to develop through training and collaborative working. The ICU patient experience could be improved if discussions about EoL took place promptly when required. This would allow forward planning to ensure that once EoL is recognised, individualised care plans can be formed. The formulation of clear guidance and care planning within ICU is necessary to ensure the transition towards EoL is efficient and effective. BJN

\section{Declaration of interest: none}

Aslakson RA, Curtis JR, Nelson JE (2014) The changing role of palliative care in the ICU. Critical Care Medicine 42(11): 2418. https://dx.doi. org/10.1097/CCM.0000000000000573

Bjurling-Sjöberg P, Wadensten B, Pöder U, Nordgren L, Jansson I (2015) Factors affecting the implementation process of clinical pathways: a mixed method study within the context of Swedish intensive care. J Eval Clin Pract 21(2): 255-61. https://dx.doi.org/10.1111/jep.12301

Campbell R (2013) An Ethical Perspective-Declining and Withdrawing Treatment. In:Tingle J, Cribb A, eds Nursing Law and Ethics. 4th edn. Blackwell Publishing, Oxford

Capuzzo M,Volta CA, Tassinati T et al (2014) Hospital mortality of adults admitted to Intensive Care Units in hospitals with and without Intermediate Care Units: a multicentre European cohort study. Crit Care 18(5). https://dx.doi.org/10.1186/s13054-014-0551-8

Chan RJ, Webster J (2013) End-of-life care pathways for improving outcomes in caring for the dying. Cochrane Database Syst Rev (11): CD008006. https://dx.doi.org/10.1002/14651858.CD008006.pub3

Chan RJ, Webster J, Bowers A (2016) End-of-life care pathways for improving outcomes in caring for the dying. Cochrane Database Syst Rev 2 : CD008006. https://dx.doi.org/10.1002/14651858.CD008006.pub4

Checkley W, Martin GS, Brown SM et al (2014) Structure, process and annual intensive care unit mortality across 69 centers: United States Critical Illness and Injury Trials Group Critical Illness Outcomes Study (USCIITGCIOS). Crit Care Med 42(2): 344-56. https://dx.doi.org/10.1097/ CCM.0b013e3182a275d7

Compassion in Dying (2015) What happened to the Liverpool Care Pathway. http://tinyurl.com/zmbscm6 (accessed 24 February 2017)

\section{KEY POINTS}

- A literature review looking at end-of-life (EoL) care in the intensive care unit (ICU) was undertaken

- Several themes emerged from the literature including difficulties with implementation and compliance, decision making and the integration of national frameworks

- There are methodological challenges with conducting research into EoL care

- The formulation of clear guidance is necessary to ensure high-quality EoL care in the ICU

Coombs M, Long T (2008) Managing a good death in critical care: can health policy help? Nurs Crit Care 13(4): 208-14. https://dx.doi.org/10.1111/ j.1478-5153.2008.00280.x

Coombs MA, Addington-Hall J, Long-Sutehall T (2012) Challenges in transition from intervention to end of life care in intensive care: a qualitative study. Int J Nurs Stud 49(5): 519-27. https://dx.doi. org/10.1016/j.ijnurstu.2011.10.019

Cox S, Handy JM, Blay A (2012) Palliative Care in the ICU.

Journal of the Intensive Care Society 13(4): 320-6. https://dx.doi. org $/ 10.1177 / 175114371201300411$

Curtis JR,Vincent J-L (2010) Ethics and end-of-life care for adults in the intensive care unit. Lancet 376(9749): 1347-53. https://dx.doi. org/10.1016/S0140-6736(10)60143-2

Curtis JR, Nielsen EL, Treece PD et al (2011) Effect of a quality-improvement intervention on end-of-life care in the intensive care unit: a randomized trial. Am J Respir Crit Care Med 183(3): 348-55. https://dx.doi. org/10.1164/rccm.201006-1004OC

Dean P, Labram A, McCarroll L, Hughes M (2010) End-of-life care in Scottish intensive care units. Journal of the Intensive Care Society 11(4): 245-9. https://dx.doi.org/10.1177/175114371001100407

Department of Health (2016) Our Commitment to You for End of Life Care: The Government Response to the Review of Choice in End of Life Care. http:// tinyurl.com/zdfdajj (accessed 24 February 2017)

Efstathiou N, Walker W (2014) Intensive care nurses' experiences of providing end-of-life care after treatment withdrawal: a qualitative study. J Clin Nurs 23(21-22): 3188-96

Glavan BJ, Engelberg RA, Downey L, Curtis JR (2008) Using the medical record to evaluate the quality of end-of-life care in the intensive care unit. Crit Care Med 36(4): 1138-46. https://dx.doi.org/10.1097/ CCM.0b013e318168f301

Hamric AB, Blackhall LJ (2007) Nurse-physician perspectives on the care of dying patients in intensive care units: collaboration, moral distress, and ethical climate. Crit Care Med 35(2): 422-9. https://dx.doi. org/10.1097/01.CCM.0000254722.50608.2D

Handy JM, Dharmadasa A, Soni N (2013) The future of the Liverpool Care Pathway in caring for the dying in intensive care. Journal of the Intensive Care Society 14(2): 100-2. https://dx.doi. org $/ 10.1177 / 175114371301400202$

Bassford C (2015) End-of-life practices in intensive care units around the world- The Ethicus II study. https://tinyurl.com/jgospng (accessed 21 February 2017)

Langley G, Schmollgruber S, Fulbrook P, Albarran JW, Latour JM (2014) South African critical care nurses' views on end-of-life decision-making and practices. Nurs Crit Care 19(1): 9-17. https://dx.doi.org/10.1111/ nicc. 12026

Leadership Alliance for the Care of Dying People (2014) One Chance To Get It Right. Improving People's Experience of Care in the Last Few Days and Hours of Life. https://tinyurl.com/mad2kql (accessed 21 February)

Monkhouse D (2013) Advances in critical care for the older patient. Reviews in Clinical Gerontology 23(2): 118-30. https://dx.doi.org/10.1017/ S0959259812000226

Morgan J (2008) End-of-life care in UK critical care units-a literature review. Nurs Crit Care 13(3): 152-61. https://dx.doi.org/10.1111/j.14785153.2008.00274.x

Morgan CK,Varas GM, Pedroza C, Almoosa KF (2014) Defining the practice of 'no escalation of care' in the ICU. Crit Care Med 42(2): 357-61. https:// dx.doi.org/10.1097/CCM.0b013e3182a276c9

National Institute for Health and Care Excellence (2011) End of life care for adults [Quality Standard S13]. https://www.nice.org.uk/guidance/qs13 (accessed 21 February 2017

National Palliative and End of Life Care Partnership (2015) Ambitions for 
Palliative and End of Life Care: A National Framework for Local Action 2015 2020. http://tinyurl.com/huxhzml (accessed 24 February 2017)

Nelson JE (2006) Identifying and overcoming the barriers to high-quality palliative care in the intensive care unit. Crit Care Med 34(11 Suppl): S324331. https://dx.doi.org/10.1097/01.CCM.0000237249.39179.B1

Penrod JD, Pronovost PJ, Livote EE et al (2012) Meeting standards of highquality intensive care unit palliative care: clinical performance and predictors. Crit Care Med 40(4): 1105-12. https://dx.doi.org/10.1097/ CCM.0b013e3182374a50

Ranse K, Yates P, Coyer F (2015) Factors influencing the provision of endof-life care in critical care settings: development and testing of a survey instrument. J Adv Nurs 71(3): 697-709. https://dx.doi.org/10.1111/ jan. 12576

Ryan L, Seymour J (2013) Death and dying in intensive care: emotional labour of nurses. End of Life Journal 3(2): 1-9. https://dx.doi.org/10.1136/ EoLjnl-03-02.1

Sprung CL, Cohen SL, Sjokvist P et al (2003) End-of-life practices in
European intensive care units: the Ethicus Study. JAMA 290(6): 790-7. https://dx.doi.org/10.1001/jama.290.6.790

Ramasamy Venkatasalu M, Whiting D, Cairnduff K (2015) Life after the Liverpool Care Pathway (LCP): a qualitative study of critical care practitioners delivering end-of-life care. J Adv Nurs 71(9): 2108-18. https://dx.doi.org/10.1111/jan.12680

Walker R, Read S (2010) The Liverpool Care Pathway in intensive care: an exploratory study of doctor and nurse perceptions. Int J Palliat Nurs 16(6): 267-73. https://dx.doi.org/10.12968/ijpn.2010.16.6.48825

Watts $T$ (2012) Initiating end-of-life care pathways: a discussion paper. J Adv Nurs 68(10): 2359-70. https://dx.doi.org/10.1111/j.13652648.2011.05924.x

Watts T (2013) End-of-life care pathways and nursing: a literature review. J Nurs Manag 21(1): 47-57. https://dx.doi.org/10.1111/j.1365-

2834.2012.01423.x

\section{CPD reflective questions}

What can be done in your area to improve end-of-life (EOL) care for patients and families?

- Does your organisation have standardised intensive care unit-specific EOL documents, and what do you think about the impact these have on the patient experience?

- Think about some of the methodological challenges to research in EOL care and how some of these might be overcome 